\title{
Performance Comparisons Of Hybrid Fuzzy-LQR And Hybrid PID-LQR Controllers On Stabilizing Double Rotary Inverted Pendulum
}

\author{
Jamilu Kamilu Adamu1, Mukhtar Fatihu Hamza ${ }^{2 *}$, and Abdulbasid Ismail Isa ${ }^{3}$ \\ ${ }^{1}$ Federal Ministry of Power, Works and Housing, Nigeria \\ ${ }^{2}$ Department of Mechatronics Engineering, Bayero University Kano, Nigeria \\ ${ }^{3}$ Department of Electrical and Electronics Engineering, Usmanu Danfodiyo University Sokoto, Nigeria
}

ABSTRACT: Double Rotary Inverted Pendulum (DRIP) is a member of the mechanical under-actuated system which is unstable and nonlinear. The DRIP has been widely used for testing different control algorithms in both simulation and experiments. The DRIP control objectives include Stabilization control, Swing-up control and trajectory tracking control. In this research, we present the design of an intelligent controller called "hybrid Fuzzy-LQR controller" for the DRIP system. Fuzzy logic controller (FLC) is combined with a Linear Quadratic Regulator (LQR). The LQR is included to improve the rules. The proposed controller was compared with the Hybrid PID-LQR controller. Simulation results indicate that the proposed hybrid Fuzzy-LQR controllers demonstrate a better performance compared with the hybrid PID-LQR controller especially in the presense of disturbances.

Key words: Double Rotary Inverted Pendulum, LQR, Fuzzy control, PID

\section{INTRODUCTION}

The Double Rotary inverted pendulum (DRIP) has double pendulums connected together and attached to a rotating arm as shown in Figure 1 (a). The plane of the two pendulums is orthogonal to the radial arm. The arm is actuated by a controlling torque with the objective of balancing the two pendulums in the inverted position. Therefore, it has three degree of freedom (DOF) [1]. The actuated joint angle can make some movement in order to stabilize the two pendulums [2]. The DRIP is a nonlinear, unstable, non-minimum phase, and under-actuated mechanical systems [3]. The schematic diagram of the experimental setup is shown in Figure 1 (b).

The DRIP systems have some applications in robotics, marine systems, aerospace systems, flexible systems, pointing control, mobile systems, and locomotive systems [4]. Moreover, at hanging position, the DRIP represents simplified industry crane model [5].

The control objectives of the DRIP can be categorized into three categories $[6,7]$ namely:

1. Swing-up control [8].

2. Stabilization control [9].

3. Trajectory tracking control [10].

The conventional PID controller is the most widely used controller in the industry due to its ease of design, simple control structure, and inexpensive cost. However, the PID controller is not suited for strongly nonlinear and uncertain systems because of it being linear controller [6]. Other control methods such as NN and FLC are introduced to overcome limitations of the classical control theory. Particularly, FLC is very effective and its power has been established in numerous applications [11].
It has been known that a slight disturbance in on output can affect the status of the other output in SIMO systems [6]. Therefore, in view of the nonlinear performance of DRIP system and its high level of disturbances and large time constant, it is not easy to achieve the desired response. Thus, the appropriate control method is the cascade control topology (Figure 2). The cascade control has the advantage of weakening the consequence of disturbances and enhance the dynamics of the entire control loop [12].

In the present study, we present the design of a hybrid controller for the DRIP system. The proposed hybrid controller includes two controllers. One is model-based (i.e. LQR) and FLC (model-free). The model inaccuracy of the system can be handled by the model-free controller. The $\mathrm{LQR}$ is included to improve the performance based on full state feedback control. The FLC is used to accommodate nonlinearity based on its IF-THEN rules. The proposed controller was compared with the Hybrid PID-LQR controller. The results found indicates that the proposed hybrid Fuzzy-LQR controllers demonstrate a better performance compared with the hybrid PID-LQR controller especially in the presence of disturbances.

\section{NONLINEAR DYNAMIC MODEL OF DRIP}

The DRIP consists of a series of two pendulums attached to a rotary arm that rotates around the motor shaft axis. It has three DOF, namely rotary arm angle $\theta$, lower pendulum angle $\alpha$, and upper pendulum angle $\gamma$. The schematic diagram of the DRIP is shown in Figure 3.

$\begin{array}{ll}\text { Received } & \text { : December 17, 2019 } \\ \text { Revised } & \text { : February 25, 2020 } \\ \text { Accepted } & \text { : Maret 08, 2020 }\end{array}$


Euler-Lagrange is used to derive the dynamic equation of the DRIP system [13].

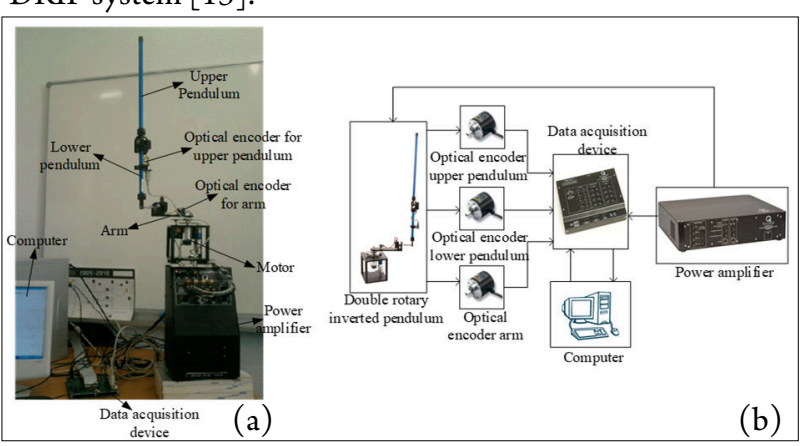

Fig. 1. Experimental setup (a) Picture (b) Schematic diagram

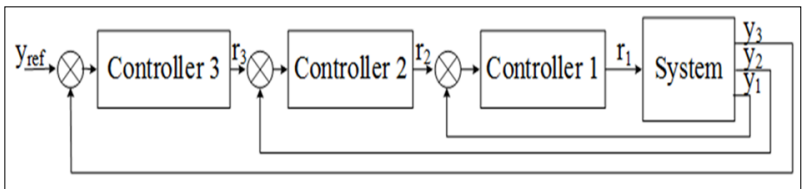

Fig. 2. General Cascade Control Structure

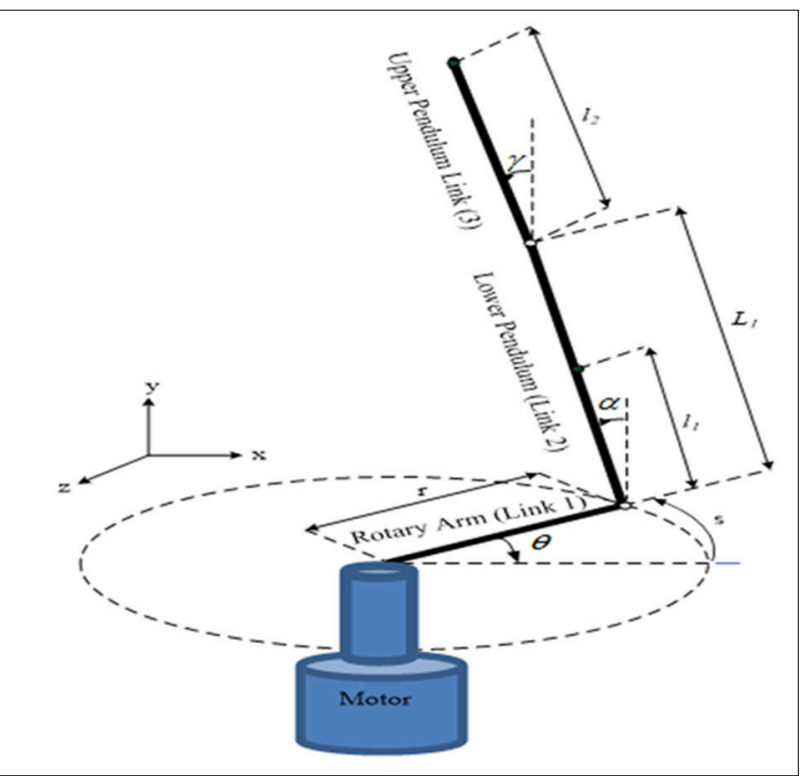

Fig. 3. Schematic Diagram of DRIP

The Euler-Lagrange Equation is given in equation (1) was used for the development of the nonlinear dynamic model of DRIP in this study[14].

$$
\begin{gathered}
\tau_{i}=\frac{d}{d t}\left[\frac{\partial \mathcal{L}}{\partial \dot{q}_{i}}\right]-\frac{\partial \mathcal{L}}{\partial q_{i}}+\frac{\partial w}{\partial \dot{q}_{i}} \\
w=\frac{1}{2} b_{i} \dot{q}_{i}^{2}=>\frac{\partial w}{\partial \dot{q}_{i}}=b_{i} \dot{q}_{i}
\end{gathered}
$$

Where $q_{i}$ are the generalize coordinates, are the generalized velocities, $\tau_{i}$ is the external force or load $\dot{q}_{i}$ vector, $\mathcal{L}$ is the Lagrangian and $w$ is the loss energy.

$$
\mathcal{L}=K-P
$$

where $K$ is the total kinetic energy of the system and $P$ is the total potential energy of the system.

Therefore, applying the Euler Lagrange Equation (1) to the Lagrangian (3) results in three coupled nonlinear equations. Euler-Lagrange equation of the motion of each link thus becomes:
For arm $(\theta)$, substituting $\theta$ in equation (1)

$$
\begin{aligned}
& \tau_{a}=\frac{d}{d t}\left[\frac{\partial \mathcal{L}}{\partial \dot{\theta}}\right]-\frac{\partial \mathcal{L}}{\partial \theta}+b_{a} \dot{\theta} \\
& \tau_{a}=\left[J_{a}+r^{2}\left(m_{1}+m_{2}\right) \ddot{\theta}\right] \\
&+r\left(m_{1} l_{1}+m_{2} L_{1}\right) \cos (\alpha) \ddot{\alpha} \\
&+m_{2} l_{2} r \ddot{\gamma} \cos (\gamma)+b_{a} \dot{\theta} \\
& \\
&-r\left(m_{1} l_{1}+m_{2} L_{1}\right) \sin (\alpha) \dot{\alpha}^{2} \\
&-m_{2} l_{2} r \sin (\gamma) \dot{\gamma}^{2}
\end{aligned}
$$

For lower pendulum $(\boldsymbol{\alpha})$, substituting $\boldsymbol{\alpha}$ in equation (1)

$$
\begin{aligned}
& 0=\frac{d}{d t}\left[\frac{\partial \mathcal{L}}{\partial \dot{\alpha}}\right]-\frac{\partial \mathcal{L}}{\partial \alpha}+b_{1} \dot{\alpha} \\
0= & r\left(m_{1} l_{1}+m_{2} L_{1}\right) \cos (\alpha) \ddot{\theta} \\
& +\left(J_{1}+m_{1} l_{1}{ }^{2}+m_{2} L_{1}^{2}\right) \ddot{\alpha} \\
& +m_{2} L_{1} l_{2} \cos (\alpha-\gamma) \ddot{\gamma} \\
& -b_{1} \dot{\alpha}+m_{2} L_{1} l_{2} \sin (\alpha-\gamma) \dot{\gamma}^{2} \\
& -g\left(m_{1} l_{1}+m_{2} L_{1}\right) \sin (\alpha)
\end{aligned}
$$

For upper pendulum $(\gamma)$, substituting $\gamma$ in equation (1)

$$
0=\frac{d}{d t}\left[\frac{\partial \mathcal{L}}{\partial \dot{\gamma}}\right]-\frac{\partial \mathcal{L}}{\partial \gamma}+b_{2} \dot{\gamma}
$$

$$
\begin{aligned}
0= & m_{2} l_{2} r \cos (\gamma) \ddot{\theta}+m_{2} L_{1} l_{2} \cos (\alpha-\gamma) \ddot{\alpha} \\
& +\left(J_{2}+m_{2} l_{2}^{2}\right) \ddot{\gamma}+b_{2} \dot{\gamma} \\
& -m_{2} L_{1} l_{2} \sin (\alpha-\gamma) \dot{\alpha}^{2} \\
& -g m_{2} l_{2} \sin (\gamma)
\end{aligned}
$$

Equations (5), (7) and (9) are three nonlinear, coupled, second-order differential equations of motion describing the dynamics equations of the DRIP system. These dynamic equations can be reduced to the following equations:

$$
\begin{aligned}
\tau_{a}= & z_{1} \ddot{\theta}+z_{2} \cos (\alpha) \ddot{\alpha}+z_{3} \ddot{\gamma} \cos (\gamma) \\
& +b_{a} \dot{\theta}-z_{2} \sin (\alpha) \dot{\alpha}^{2}-z_{3} \sin (\gamma) \dot{\gamma}^{2} \\
0= & z_{2} \cos (\alpha) \ddot{\theta}+z_{4} \ddot{\alpha}+z_{5} \cos (\alpha-\gamma) \ddot{\gamma} \\
& +b_{1} \dot{\alpha}+z_{5} \sin (\alpha-\gamma) \dot{\gamma}^{2}-z_{7} \sin (\alpha) \\
0= & z_{3} \cos (\gamma) \ddot{\theta}+z_{5} \cos (\alpha-\gamma) \ddot{\alpha}+z_{6} \ddot{\gamma} \\
& +b_{2} \dot{\gamma}-z_{5} \sin (\alpha-\gamma) \dot{\alpha}^{2}-z_{8} \sin (\gamma)
\end{aligned}
$$

Where:

$$
\begin{gathered}
Z_{1}=J_{a}+r^{2}\left(m_{1}+m_{2}\right) \\
Z_{2}=r\left(m_{1} l_{1}+m_{2} l_{2}\right) \\
Z_{3}=m_{2} l_{2} \\
Z_{4}=J_{1}+m_{1} l_{1}^{2}+m_{2} l_{1}^{2} \\
Z_{5}=L_{1} l_{2} m_{2} \\
Z_{6}=J_{2}+m_{2} l_{2}^{2} \\
Z_{7}=g\left(m_{1} l_{1}+m_{2} l_{1}\right) \\
Z_{8}=g m_{2} l_{2}
\end{gathered}
$$

The torque at the load shaft from an applied motor torque can be express as: 
The torque at the load shaft from an applied motor torque can be express as:

$$
\tau_{m}(t)=\frac{\eta_{g} K_{g} \eta_{m} k_{t}\left(V_{m}(t)-K_{g} k_{m} \dot{\theta}(t)\right)}{R_{m}}
$$

The value of the torque for the system under consideration can be calculated using equation (21) below.

$$
\tau_{a}=0.117238 v-0.063 \dot{\theta} \mathrm{Nm}
$$

\section{SYSTEM SPECIFICATIONS}

The system specification and their description are given in Table 1 (15).

\begin{tabular}{|c|c|c|c|}
\hline Symbol & Description & Value & Unit \\
\hline$J_{a}$ & $\begin{array}{l}\text { Moment of inertia about } \\
\text { the center of mass for } \\
\text { rotary arm }\end{array}$ & 0.0041 & $\mathrm{~kg} \cdot \mathrm{m}^{2}$ \\
\hline$J_{I}$ & $\begin{array}{l}\text { Moment of inertia about } \\
\text { the center of mass for } \\
\text { upper Pendulum }\end{array}$ & 0.00032 & kg.m ${ }^{2}$ \\
\hline$J_{2}$ & $\begin{array}{l}\text { Moment of inertia about } \\
\text { the center of mass for } \\
\text { lower Pendulum }\end{array}$ & 0.0012 & $\mathrm{~kg} \cdot \mathrm{m}^{2}$ \\
\hline$r$ & $\begin{array}{l}\text { Length of rotary arm from } \\
\text { pivot to tip }\end{array}$ & 0.2159 & $\mathrm{M}$ \\
\hline$L_{1}$ & $\begin{array}{l}\text { Length of the lower } \\
\text { pendulum from pivot to } \\
\text { tip }\end{array}$ & 0.2 & M \\
\hline$I_{1}$ & $\begin{array}{l}\text { Length of the lower } \\
\text { pendulum from pivot to } \\
\text { centre of mass }\end{array}$ & 0.097 & M \\
\hline$I_{2}$ & $\begin{array}{l}\text { Length of the upper } \\
\text { pendulum from pivot to } \\
\text { centre of mass }\end{array}$ & 0.156 & M \\
\hline$b_{a}$ & $\begin{array}{l}\text { Motor arm's viscous } \\
\text { damping coefficient of the. } \\
\text { Upper Pendulum's }\end{array}$ & 0.0024 & $\begin{array}{l}\mathrm{N} . \mathrm{m} / \\
(\mathrm{rad} / \mathrm{s})\end{array}$ \\
\hline$b_{1}$ & $\begin{array}{l}\text { viscous damping } \\
\text { coefficient as seen at the } \\
\text { pivot axis }\end{array}$ & 0.0024 & $\begin{array}{l}\mathrm{N} \cdot \mathrm{m} / \\
(\mathrm{rad} / \mathrm{s})\end{array}$ \\
\hline$b_{2}$ & $\begin{array}{l}\text { Lower Pendulum's } \\
\text { viscous damping } \\
\text { coefficient as seen at the } \\
\text { pivot axis }\end{array}$ & 0.0024 & $\begin{array}{l}\mathrm{N} . \mathrm{m} / \\
(\mathrm{rad} / \mathrm{s})\end{array}$ \\
\hline$V_{\text {nom }}$ & $\begin{array}{l}\text { Motor nominal input } \\
\text { voltage }\end{array}$ & 6.0 & $\mathrm{~V}$ \\
\hline$R_{m}$ & Motor armature resistance & 2.6 & $\Omega$ \\
\hline$\eta_{m}$ & Motor efficiency & 0.63 & \\
\hline$\eta_{g}$ & Gear efficiency & 0.9 & \\
\hline$K_{g}$ & Total gear ratio & 70 & \\
\hline$k_{m}$ & Back-emf constant & 0.00768 & $\begin{array}{c}\mathrm{V} / \\
(\mathrm{rad} / \mathrm{s})\end{array}$ \\
\hline$k_{t}$ & Motor torque constant & 0.00768 & N.m \\
\hline
\end{tabular}

Table 1 SRV02 DRIP Specifications

\section{PROPOSED CONTROL STRATEGY}

The proposed control method consists of a combination of PID, LQR and FLC. This section explains the technicalities in their individual control and their hybrid control.

\subsection{Proportional Integral Derivative (PID) Controller}

The PID controller sigmatic diagram is shown in figure 3. It is a feedback controller that is based on the error (e) between the desired point and measured process value. Three parameters are designed in the PID controller and each parameter has an effect on the error [16].

Defining " $u$ " as the controller output, the final PID algorithm is of the form [17]:

$$
\begin{aligned}
u(t) & =K_{P} e(t)+K_{I} \int_{0}^{t} e(\tau) d \tau+K_{D} \frac{d e(t)}{d t} \\
& =K_{P} e(t)+\frac{1}{T_{I}} \int_{0}^{t} e(\tau) d \tau+T_{D} \frac{d e(t)}{d t}
\end{aligned}
$$

Where: $K_{p}=$ proportional gain, $K_{I}=$ integral gain, $K_{D}$ derivative gain, $T_{I}=$ integral time constant and $T_{D}=$ the derivative time constant. These are the tuning parameters used to design a PID controller that is varied to get an optimum response.

\subsection{Linear Quadratic Regulator (LQR)}

The LQR is an optimal state feedback controller. It is used to obtain the optimal performance of the system by minimizing the cost function that relates the state vector and control input vector. The $\mathrm{LQR}$ method is a powerful technique for designing controllers for complex systems that have stringent performance requirements and it seeks to find the optimal controller that minimizes a given cost function. The conventional LQR problem is to obtain the control input $u$ as follows [18]:

$$
u(t)=-k x(t)
$$

which minimizes the following cost function.

$$
J(u)=\int_{0}^{t_{f}}\left(x^{T}(t) Q x(t)+u^{T}(t) R u(t)\right) d t
$$

where $\mathrm{Q}$ and $\mathrm{R}$ are square matrices and are always positive semidefinite. The matrices $\mathrm{Q}$ and $\mathrm{R}$ scale the relative contributions of the terms of the quadratic forms $x^{T}(t)_{X}(t)$ and $u^{T}(t) u(t)$ in the integral respectively [19]. Thus the elements of $\mathrm{Q}$ penalizes the states $\mathrm{x}$ and $\mathrm{R}$ penalizes the control $u$ in the performance index. For this reason $\mathrm{Q}$ and $\mathrm{R}$ are called weighting matrices $[20[. J$ is always a scalar quantity.

The linear control law given by Equation (24) is the optimal control law. Therefore, if the unknown elements of the matrix $\mathrm{K}$ are determined so as to minimize the performance index (25), then $u(t)=-k x(t)$ is optimal for any initial state $x(0)[20]$. To obtain the optimal solution for the control signal a Pontryagin principle is applied to minimize the performance index. This minimization is based on the Hamiltonian equation.

\subsection{Closed-loop Optimal Control Model}

To formulate the optimal control loop model, it means writing the optimal control law as a function of state $\mathrm{X}(\mathrm{t})$ and costate $\lambda(t)$. The state $X(t)$ is a vector consisting set of variables given in the following equation [21]:

$X(t)=\left[\begin{array}{llll}x_{1}(t) & x_{2}(t) & x_{3}(t) & x_{4}(t)\end{array}\right]$ 
Let us assume a transformation

$$
\lambda(t)=P(t) X(t)
$$

from functional analysis theory of normed linear space, $\lambda(t)$ lies in the "dual space" of $X(t)$, which is the space consisting of all continuous linear functional of $X(t)$ [21].

Where $\mathrm{P}(\mathrm{t})$ is called the Riccati coefficient matrix or simply Riccati matrix or Riccati coefficient.

$$
u(t)=-R^{-1} B^{T} P(t) X(t)
$$

which is now negative feedback of the state $\mathrm{x}(\mathrm{t})$.

Let $k=R^{-1} B^{T} P(t)$

Therefore

$$
u(t)=-k x(t)
$$

The aim is to find a control $u(t)$ over $t_{o} \leq t \leq t_{f}$ which for any $x_{0} \in R_{n}$ minimizes the cost function. Where is called Kalman gain and $\mathrm{P}(\mathrm{t})$ which is nxn symmetric, positive definite matrix is the solution of the differential Riccati equation (DRE). This equation can be clearly seen in Figure 4.

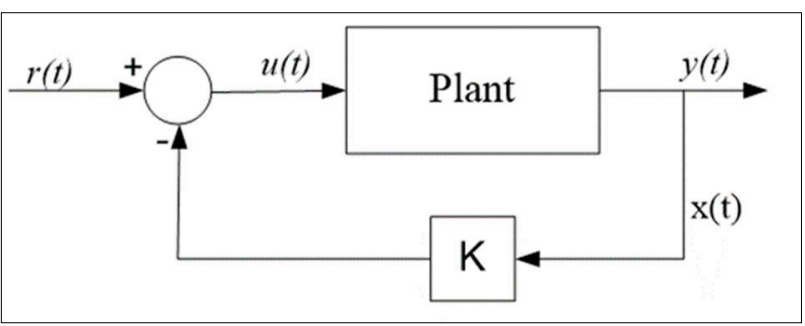

Fig. 4. LQR Control System

The Algebraic Riccati Equation (ARE) is

$$
\dot{P}+P A+A^{T} P-P B R^{-1} B^{T} P+Q=0
$$

As time $t_{f}$ approaches infinity, the optimal control law becomes constant and therefore $\dot{P}=O$. In this case, equation (29) becomes

$$
P A+A^{T} P-P B R^{-1} B^{T} P+Q=0
$$

The major concern is to develop a stable linear feedback control law defined in (28) that can minimize the performance index $\mathrm{J}$.

\subsection{Fuzzy Logic Control}

Fuzzy logic was introduced by Lotfi Zadeh in 1965, it has many successful applications especially in control [22]. FLC offers a proper method for manipulating, representing, and implementing a heuristic knowledge of human about how to control a system [6].

\subsection{Component of Fuzzy Logic Controller}

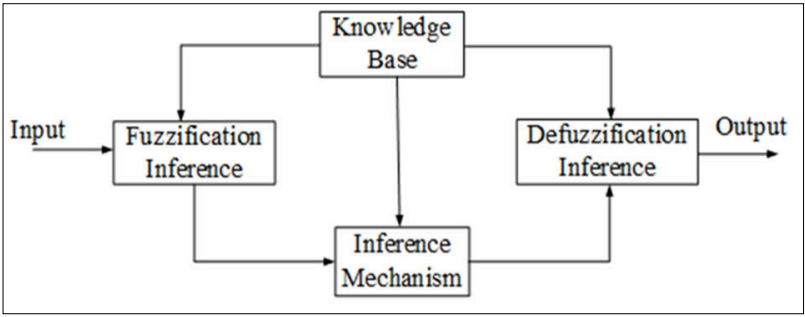

Fig. 5. Fuzzy controller block diagram

FLC has four main components, namely: fuzzification interface, rule-base, inference mechanism, and defuzzifica- tion interface as shown in Figure 5. The detailed explanation of these four FLC blocks can be found in Hamza et al.[4].

The fuzzy rule can be represented by the following fuzzy relation:

$\mathrm{R}$ : If $\mathrm{a}$ is $\mathrm{X}$ then $\mathrm{b}$ is $\mathrm{Y}$., or in abbreviated form as

$R: X \longrightarrow Y$ or $R=X \longrightarrow Y$

$\mathrm{R}$ can be viewed as a fuzzy set with a two-dimensional membership function

$$
\mu_{R}(b)=f\left(\mu_{X}(a), \mu_{Y}(b)\right)
$$

The detailed explanations on the rest of the components can be found in [23].

\subsection{Hybrid Fuzzy-LQR Controller}

From the input/output point of view, the hybrid Fuzzy-LQR controller is analogous to a conventional PID controller. As such the hybrid Fuzzy-LQR controller is considered as an alternative to conventional PID controllers [24]. There are three types of hybrid Fuzzy-LQR controller namely: fuzzy gain scheduling type, fuzzy direct-action type, and hybrid type hybrid Fuzzy-LQR controller type [25]. In this study, double input direct action type and hybrid type hybrid Fuzzy-LQR controllers are considered.

The hybrid Fuzzy-LQR controller (Figure 6) is constructed by the combination of a two-input direct action hybrid Fuzzy-LQR controller and a conventional PID controller [25]. The output of the hybrid Fuzzy-LQR controller is defined as:

$$
u=\alpha U+\beta \int U d t+K
$$

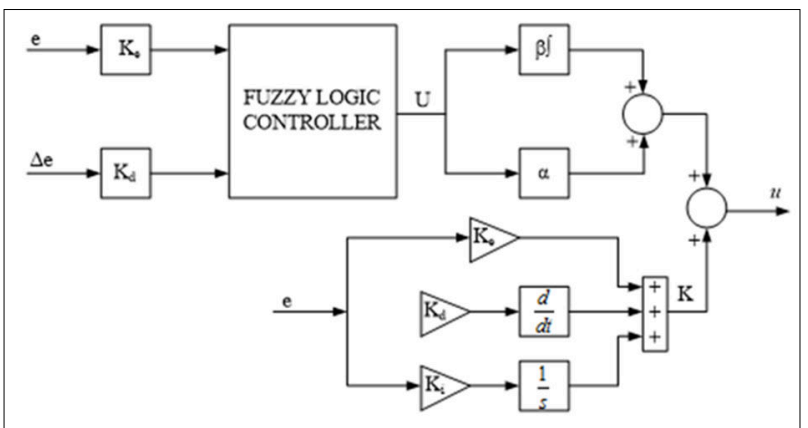

Fig. 6. hybrid Fuzzy-LQR controller

The DRIP is a single input multiple output (SIMO) system. Since PID has the limitation of controlling only one output [7], three PID/PID-Fuzzy controllers in cascade topology combined with $\mathrm{LQR}$ controller is proposed in the present study as shown in Figure 7.

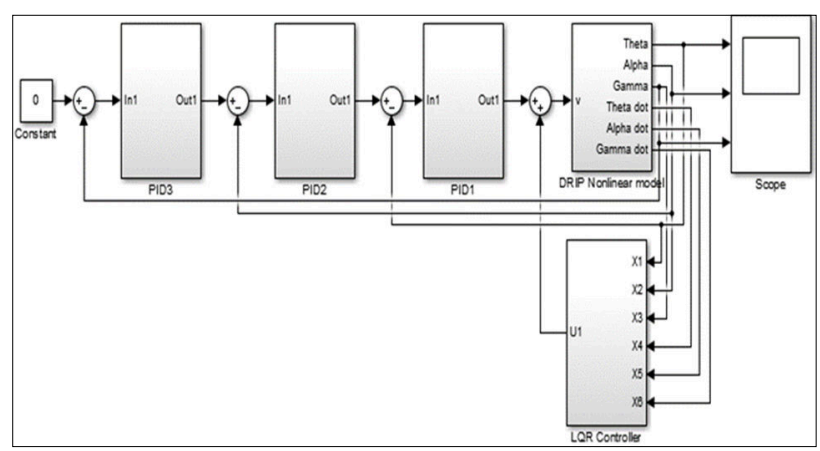

Fig. 7. Hybrid Cascade PID/LQR Controller Structure for Double Rotary Inverted Pendulum 


\subsection{PID Controller Design}

The conventional PID can only control one output. However the system under consideration has three outputs to be controlled; arm angular position, lower pendulum angle and upper pendulum angle. Therefore, three PID controllers in the cascade are designed with each controlling one output. The manual tuning was used to obtain the parameters of the PID for each of the links. After iterative manual tuning, the values of PID gains for each of the three links of DRIP are presented in Table 2.

Table 2 PID controller gains

\begin{tabular}{lccc}
\hline \multicolumn{1}{r}{ PID Gains } & KP & KI & KD \\
\hline Arm & 0.09 & 0.37 & 0.29 \\
Lower Pendulum & 0.96 & 0.00022 & 0.009 \\
Upper Pendulum & 1.019 & 0.0001 & 0.09 \\
\hline
\end{tabular}

\subsection{LQR Controller Design}

LQR measures the system's states and stabilizes it using full state feedback. To design a state feedback control $u=-k x$ for system stabilization, the choosing of $\mathrm{K}$ is a trade-off between the control effort and transient response [26].

The weighting matrices $Q$ and $R$ are very important constituents of LQR optimal control process. The selection of matrices $\mathrm{Q}$ and $\mathrm{R}$ is normally based on an iterative procedure using experience and understanding of the physical problems involved to get the desired response. The number of elements of $\mathrm{Q}$ and $\mathrm{R}$ matrices depends on the number of state variables $(n)$ and the number of the input variable $(m)$, respectively [21].

We have used the LQR function in Matlab to determine the value of the vector $K$ that determines the feedback control law as follows:

$$
\begin{aligned}
& \mathrm{K}=\left[\begin{array}{lllll}
0.3162 & -46.4165 & 61.2213 & 0.2169 & -0.8726
\end{array}\right. \\
& \text { 6.6182] }
\end{aligned}
$$

For the pendulum problem under consideration, with two inputs and five linguistic values for each of these, there are at most $72=49$ possible rules. Since the input to the FLC is only two for the purpose of this research, the convenient way to list all possible rules is to use a tabular representation as shown in Table 3.

Table 3 Fuzzy Rules for DRIP

\begin{tabular}{ccccccccc}
\hline & & \multicolumn{7}{c}{ Change-In-Error } \\
\cline { 3 - 8 } & & NB & NM & NS & Z & PS & PM & PB \\
\hline & NB & NB & NB & NB & NB & NM & NS & Z \\
E & NM & NB & NB & NB & NM & NS & Z & PS \\
r & NS & NB & NB & NM & NS & Z & PS & PM \\
r & Z & NB & NM & NS & Z & PS & PM & PB \\
o & PS & NM & NS & Z & PS & PM & PB & PB \\
r & PM & NS & Z & PS & PM & PB & PB & PB \\
& PB & Z & PS & PM & PB & PB & PB & PB \\
\hline
\end{tabular}

\subsection{Hybrid Fuzzy-LQR controller Structure}

The standard hybrid Fuzzy-LQR controller is constructed by choosing the inputs to be an error (e) and derivative of error $(\Delta \mathrm{e})$ as shown and the output is the control signal (u). As indicated in Meshkov \& Sokolov [79], among the three categories of hybrid Fuzzy-LQR controller structure, double input type is the most robust structure for unstable pole systems. As can be seen from Figure 8, the handled hybrid Fuzzy-LQR controller structure has two input and two output scaling factors. The input SFs Ke (for error (e)) and $\mathrm{Kd}$ (for the change of error $(\Delta \mathrm{e})$ ). While the FLC output (U) is mapped onto the respective actual output (u) domain by output scaling factors $\beta$ and $\alpha$.

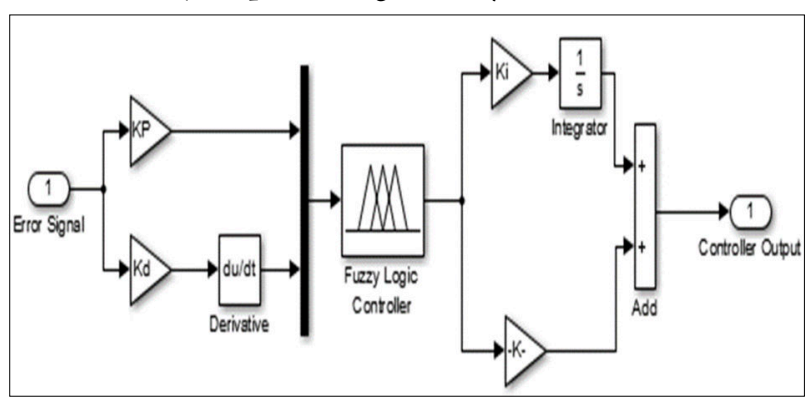

Fig. 8. Internal Structure of hybrid Fuzzy-LQR controller

\section{RESULTS AND DISCUSSIONS}

The results found in our simulation experiments are presented in this section. This includes the comparisons of the stabilization capability of hybrid PID and hybrid Fuzzy-LQR controller. The disturbance rejection ability of the proposed controllers was also presented.

\subsection{Stabilization Control Using Cascade Hybrid PID- LQR and hybrid Fuzzy-LQR controllers}

In this section, stabilization control of DRIP using cascade hybrid PID and hybrid Fuzzy-LQR controller has been analysed. Figure 9,10 and 11 shows the stabilization results for the arm angle, lower pendulum angle and upper pendulum angle respectively. It can be seen that for the arm stabilization, the hybrid Fuzzy-LQR controller outperformed the hybrid PID-LQR in terms of the performance indices considered. This is the same for both upper and lower pendulums. However, the rise time is lower for the hybrid PID-LQR for both upper and lower pendulums. The performance indices considered are rise time, settling time, overshoot, undershoot and steady-state error.

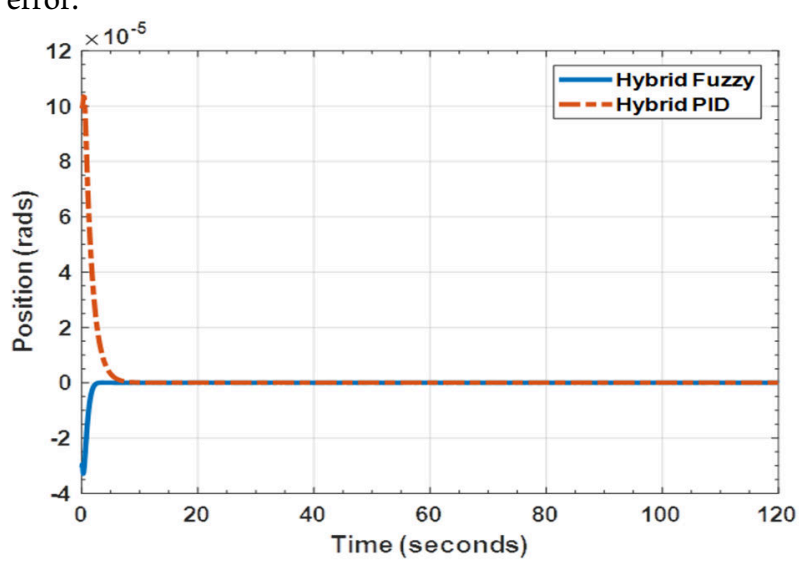

Fig. 9. Arm angle 


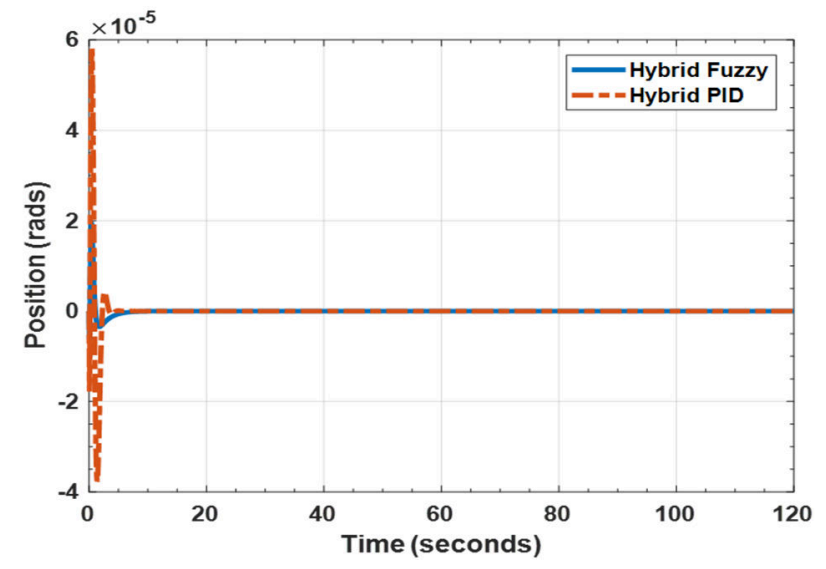

Fig. 10. Lower pendulum angle

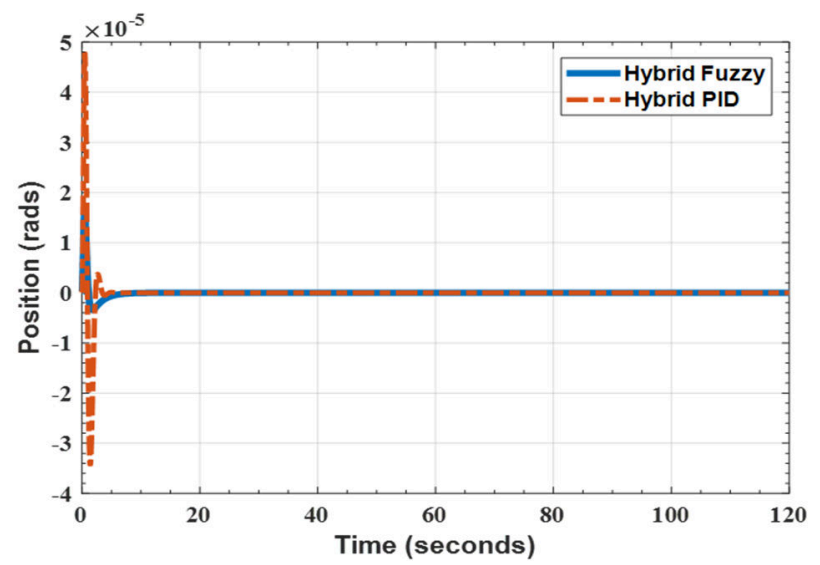

Fig. 11. Upper pendulum angle

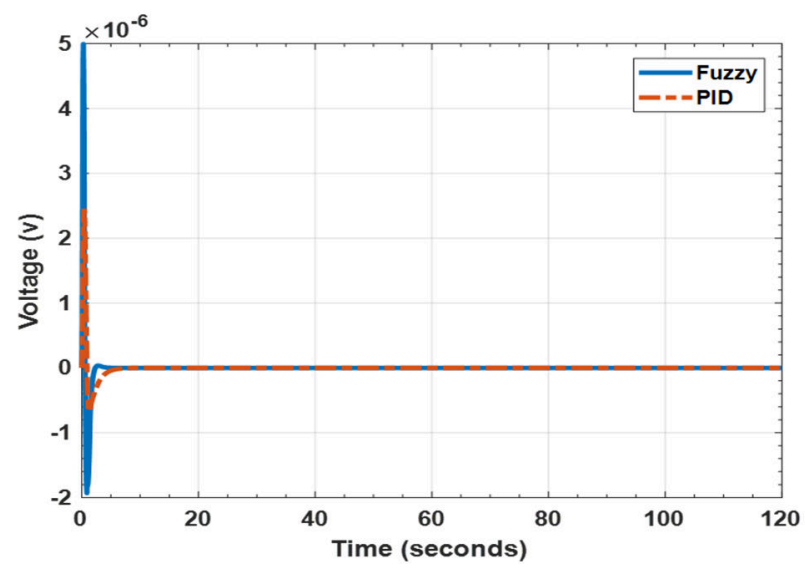

Fig. 12. Outputs of the outer controllers

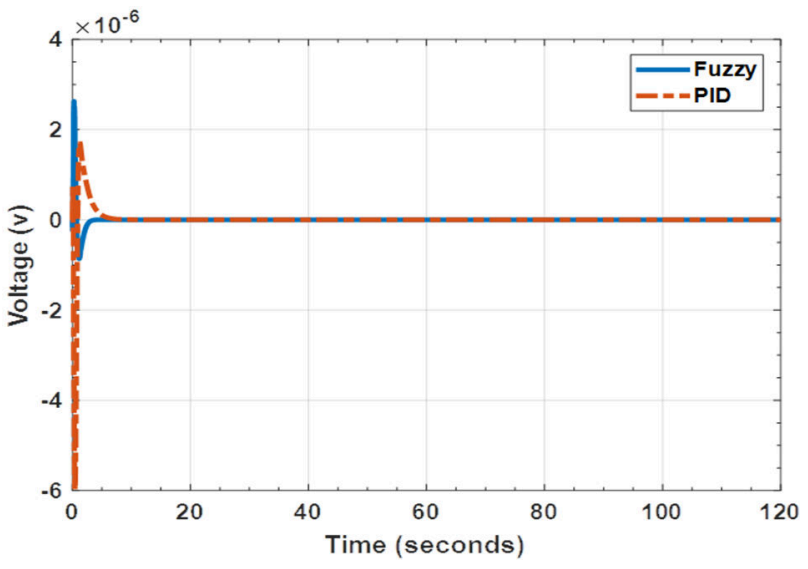

Fig. 13. Outputs of the inner controllers

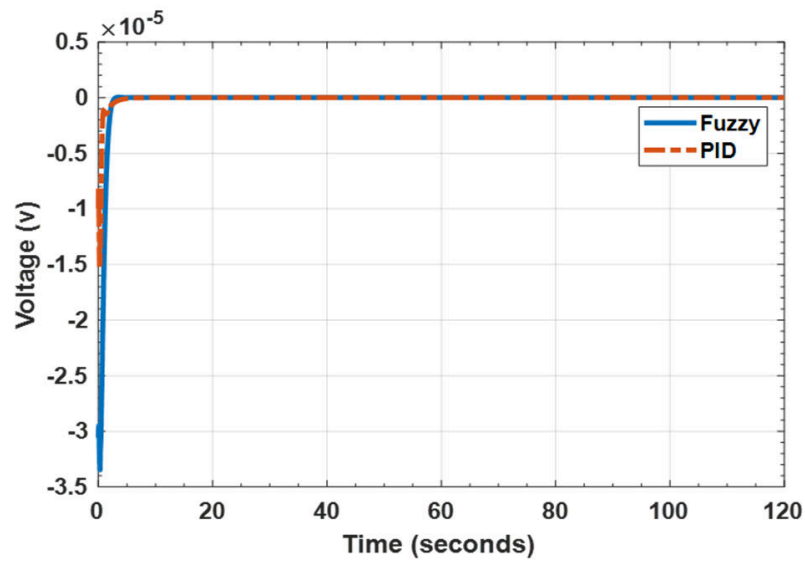

Fig. 14. Outputs of the innermost controllers

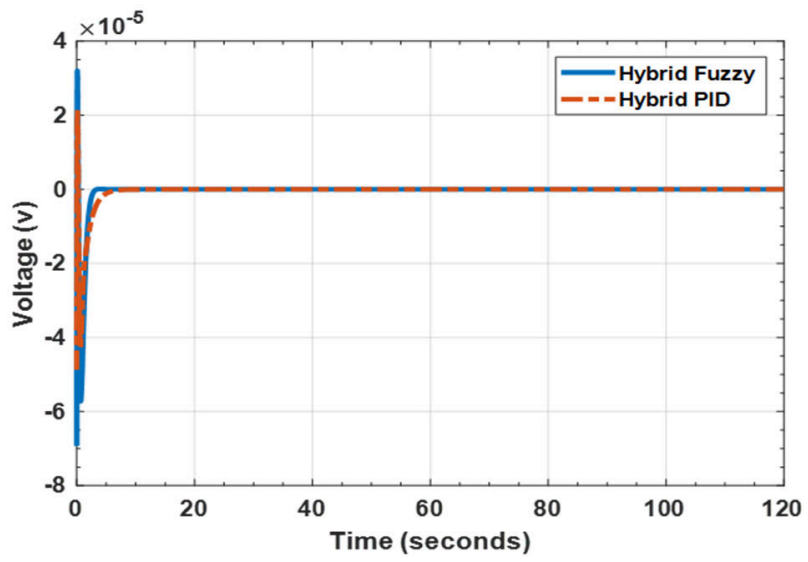

Fig. 15. Outputs of the hybrid controllers

Table 4 Performance Indices

\begin{tabular}{llccccc}
\hline & \multicolumn{5}{c}{ Controllers } & \multicolumn{4}{c}{ System Output Characteristics } \\
\cline { 3 - 6 } & & $\begin{array}{c}\text { Rise Time } \\
(\mathrm{s})\end{array}$ & $\begin{array}{c}\text { Settling } \\
\text { Time }(\mathrm{s})\end{array}$ & $\begin{array}{c}\text { Overshoot } \\
(\%)\end{array}$ & $\begin{array}{c}\text { Undershoot } \\
(\%)\end{array}$ & $\begin{array}{c}\text { Steady State } \\
\text { Error }\end{array}$ \\
\hline \multirow{2}{*}{ Arm } & Hybrid PID-LQR & 0.263 & 5.142 & 0.538 & 1.302 & 0.00012 \\
& Hybrid Fuzzy-LQR & 0.201 & 2.321 & 0.000 & -0.00023 & 0.000 \\
Lower Pendulum & Hybrid PID-LQR & 0.193 & 3.921 & 0.557 & 2.083 & -0.000033 \\
& Hybrid Fuzzy-LQR & 0.300 & 2.893 & 0.00074 & 0.00000101 & 0.000 \\
\multirow{2}{*}{ Upper Pendulum } & Hybrid PID-LQR & 0.200 & 3.443 & 0.194 & 0.714 & -0.000015 \\
& Hybrid Fuzzy-LQR & 0.311 & 3.028 & 0.00012 & 0.00000107 & 0.000 \\
\hline
\end{tabular}


Figure 12, 13, 14 and 15 shows output signals of the outer, inner and innermost controllers respectively. The effort put by individual controllers trying to stabilize the system can be seen. These results indicates that after the system stabilised the controller effort becomes almost zero since there are no disturbances.

Rise time, setteling time, overshoot, undershoot and steady state error are the five performance indeces used in this research. Table 4 shows the performance indices for arm, lower pendulum and upper pendulum. This table compare the results for hybrid PID-LQR and hybrid hybrid Fuzzy-LQR controller. It can be seen that the Hybrid Fuzzy-LQR have better result in settling time, overshoot and steady state error compare with Hybrid PID-LQR.

5.2 Disturbances rejection analysis for cascade hybrid PID and cascade hybrid hybrid Fuzzy-LQR controller

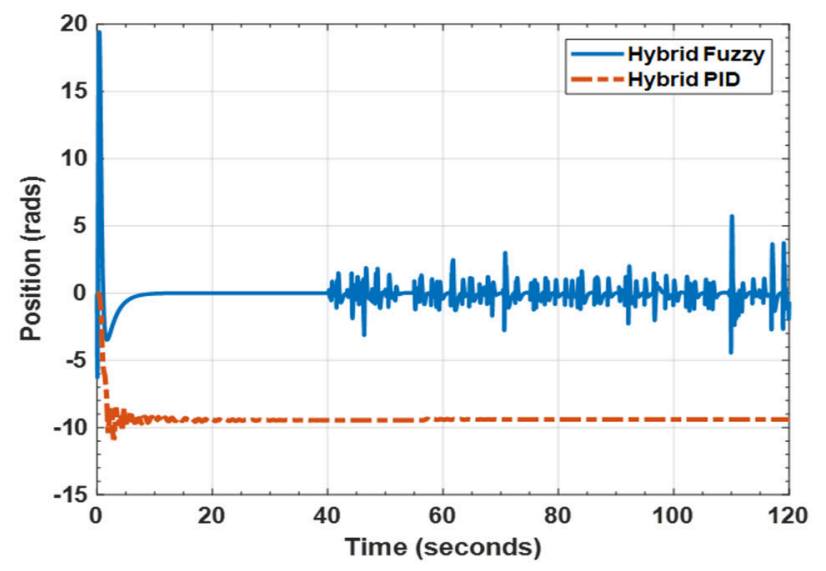

Fig. 16. Lower pendulum angle

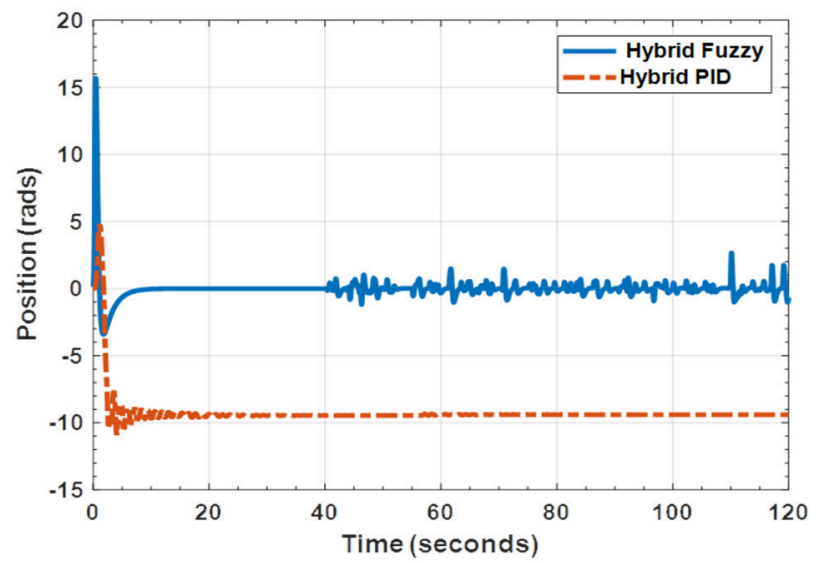

Fig. 17. Upper pendulum angle

The disturbance is introduced to the system to test for the performances and robustness of the proposed controllers. The white noise of 0.01 power parameter value is added to the process output (feedback) after stabilised at 40 seconds. Figure 16 and 17 shows the simulation results of the lower pendulum angle, upper pendulum angle and arm angle. It can be seen from Figure 16 and 17 that the proposed hybrid Fuzzy-LQR controller is able to control both upper and lower pendulums to remain at stabilization position with some oscillation. This oscillation is due to the introduced disturbance. On the other hand, from the same Figure we can see that as soon as the disturbance is introduced, the hybrid PID-LQR cannot control the upper and lower pendulums. Therefore the upper and lower pendulums fail to remain in the stabilised position. These results indicate the effectiveness and robustness of the hybrid Fuzzy-LQR controllers in the presence of disturbances as compared to the hybrid PID.

It will be good for the signals for the individual controllers in the presence of disturbances. This can help to appreciate the effort of the individual controllers in the presence of disturbances compared with that at no disturbances. Figures 18-25. It can be seen that both controllers are trying very hard to control the system to remain at stabilised position.

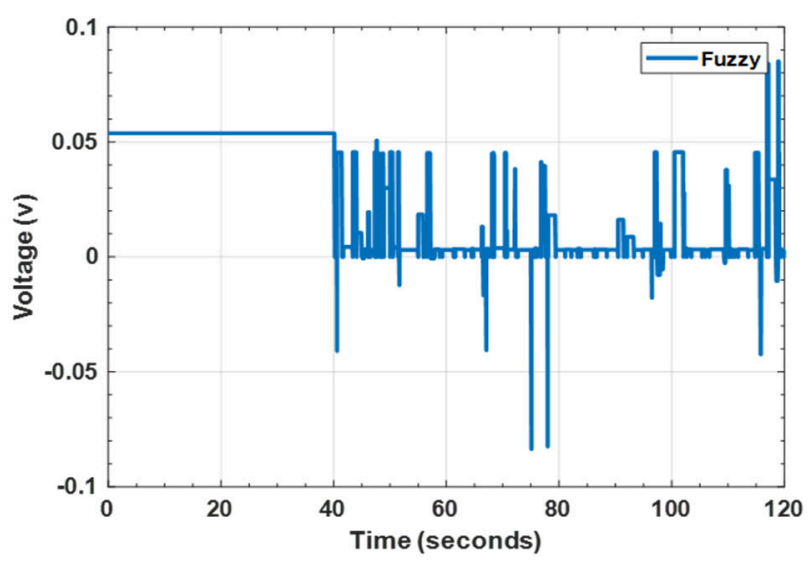

Fig. 18. Output of the outer fuzzy controller

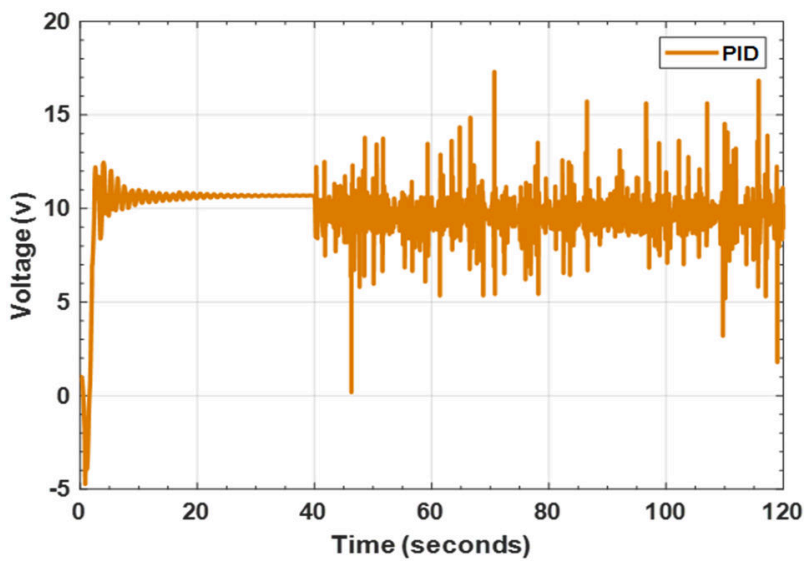

Fig. 19. Output of the outer PID controller

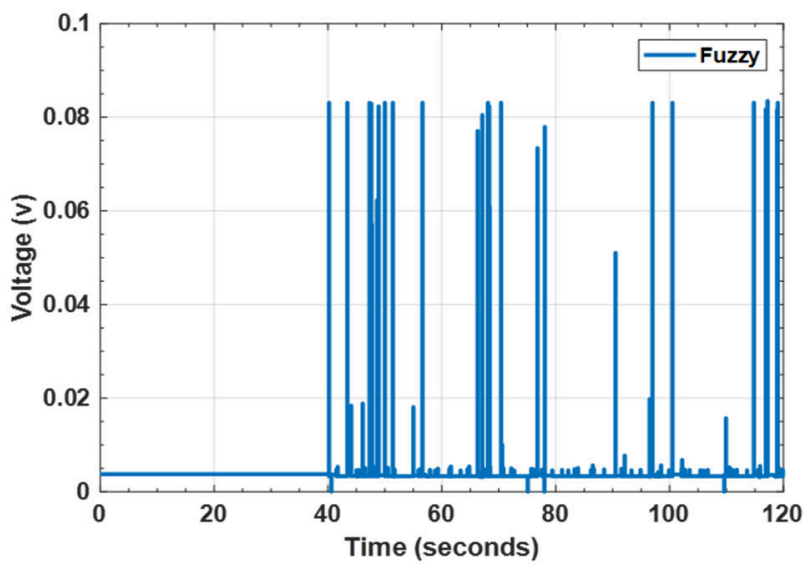

Fig. 20. Output of the inner fuzzy controller 


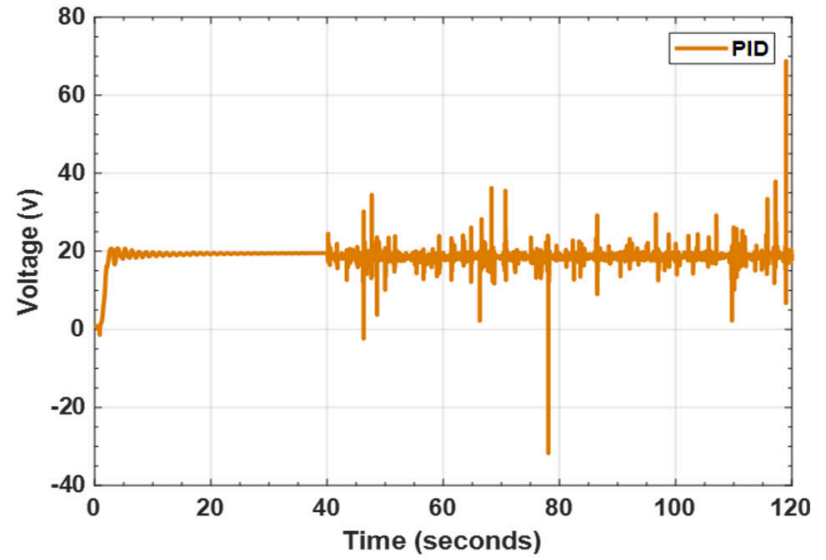

Fig. 21. Output of the inner PID controller

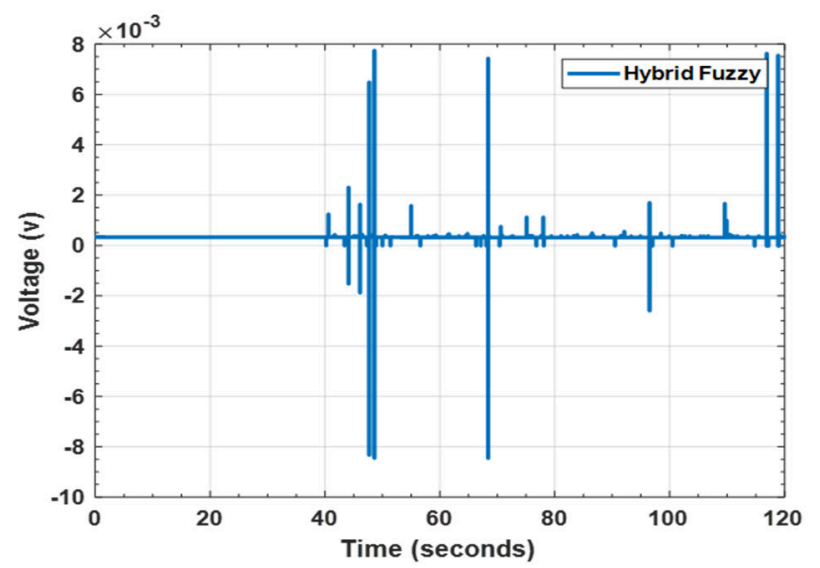

Fig. 22. Output of the innermost fuzzy controller

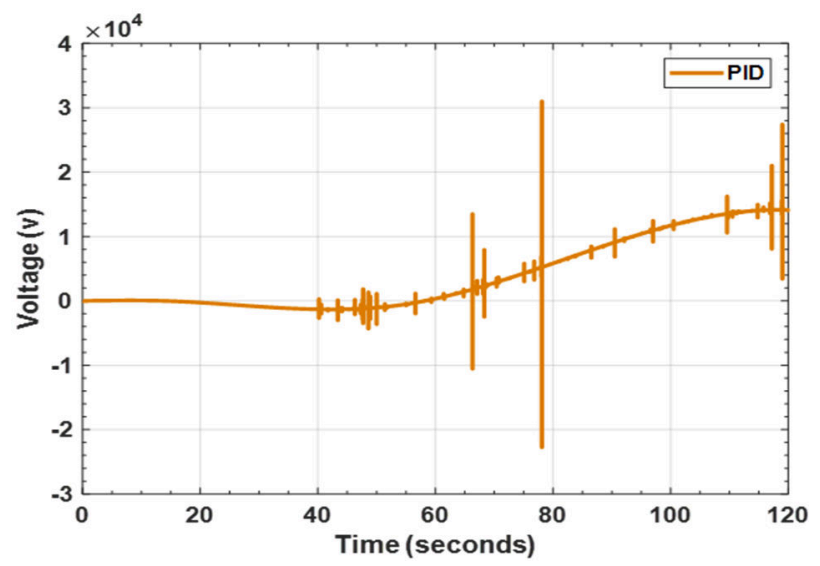

Fig. 23. Output of the innermost PID controller

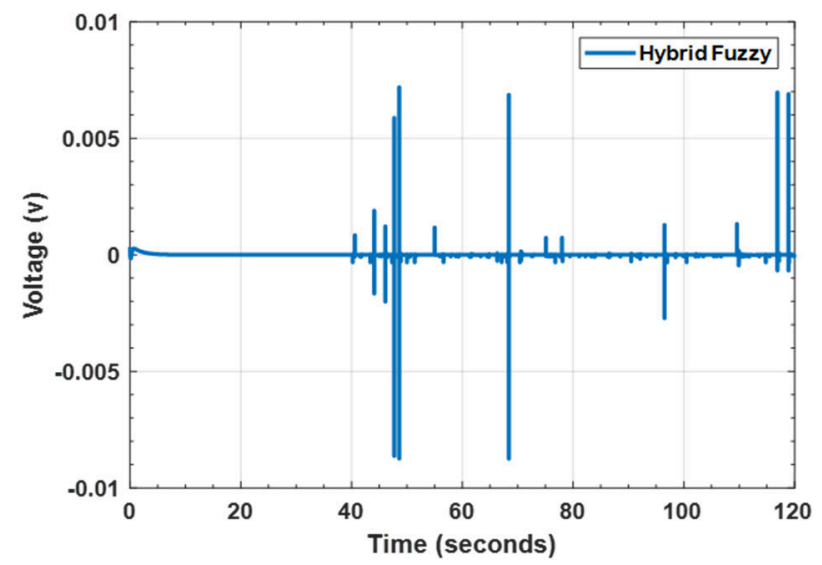

Fig. 24. Outputs of the hybrid fuzzy controllers

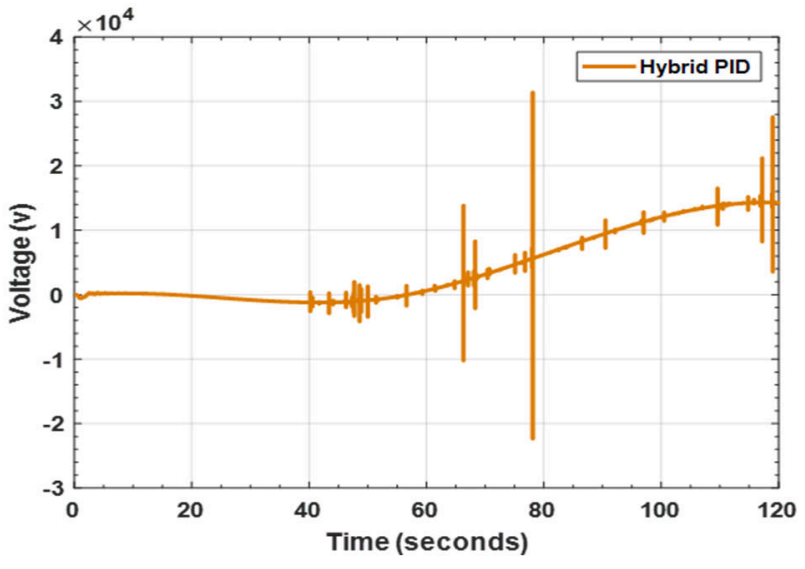

Fig. 25. Outputs of the hybrid PID controllers

\subsection{Control Effort}

The control effort is the amount of energy necessary for the controller to perform its duty. In practical control systems, we often need to minimize the control effort so as to achieve control objectives under limitations in the system under consideration. Tables 5 presents the optimized control efforts of the designed control algorithm considered in the present study for controlling the DRIP with and without disturbance respectively.

Table 5 Control Effort

\begin{tabular}{lcc}
\hline \multicolumn{1}{c}{ Controller } & $\begin{array}{c}\text { Hybrid Fuzzy- } \\
\text { LQR controller }\end{array}$ & $\begin{array}{c}\text { Hybrid } \\
\text { PID }\end{array}$ \\
\hline $\begin{array}{l}\text { Hybrid controller output } \\
\text { (v) }\end{array}$ & 4.5 & 15.12 \\
Controller 1 output (v) & 17.72 & 55.86 \\
Controller 2 output (v) & 17.78 & 55.88 \\
Controller 3 output (v) & 17.89 & 55.90
\end{tabular}

\section{CONCLUSIONS AND RECOMIMENDATIONS}

The main aim of this study is to examine the application of hybrid HYBRID FUZZY-LQR CONTROLLER and hybrid PID in cascade topology on nonlinear stabilization of DRIP. The proposed hybrid Fuzzy-LQR controller and PID were evaluated in cascade structures for stabilization control of DRIP system.

It has been demonstrated that in the absence of disturbance, both the controllers were able to stabilize the DRIP. Though, hybrid Fuzzy-LQR controller performs better than the hybrid PID controllers. However, in the presence of disturbance hybrid Fuzzy-LQR controller was able to reject the disturbance whereas hybrid PID has demonstrated very poor performance. This implies hybrid Fuzzy-LQR controller has greatly outperformed hybrid PID. Consequently, hybrid Fuzzy-LQR controller control strategy can be regarded as a promising strategy for controlling highly nonlinear, unstable, non-minimum phase and under-actuated mechanical systems especially in the presence of noise and disturbances. 


\section{AUTHOR INFORMATION}

\section{Corresponding Author}

*Email: mfhamza.mct@buk.edu.ng

\section{ORCID}

Mukhtar Fatihu Hamza : 0000-0002-7111-5767

\section{REFERENCES}

[1] Tang Teng Fong, Chong Shin Horng, Pang Kee Kiat. Stabilisation of a rotary inverted pendulum system with double-PID and LQR control: experimental verification. International Journal of Automation and Control. 2020; 14(1)

[2] Pakdeepattarakorn P., Thamvechvitee P., Songsiri J., Wongsaisuwan M., Banjerdpongchai D.. Dynamic models of a rotary double inverted pendulum system. 2004 IEEE Region 10 Conference TENCON 2004.. null.

[3] Hamza Mukhtar Fatihu, Yap Hwa Jen, Choudhury Imtiaz Ahmed, Isa Abdulbasid Ismail, Zimit Aminu Yahaya, Kumbasar Tufan. Current development on using Rotary Inverted Pendulum as a benchmark for testing linear and nonlinear control algorithms. Mechanical Systems and Signal Processing. 2019; 116

[4] Hamza Mukhtar Fatihu, Yap Hwa Jen, Choudhury Imtiaz Ahmed, Isa Abdulbasid Ismail. Application of Kane's Method for Dynamic Modeling of Rotary Inverted Pendulum System. First International Conference on Micro and Nano Technologies, Modelling and Simulation. 2016;27-32.

[5] Moreno-Valenzuela Javier, Aguilar-Avelar Carlos. Motion Control of Underactuated Mechanical Systems. Springer; 2018.

[6] Hamza Mukhtar Fatihu, Yap Hwa Jen, Choudhury Imtiaz Ahmed. Genetic Algorithm and Particle Swarm Optimization Based Cascade Interval Type 2 Fuzzy PD Controller for Rotary Inverted Pendulum System. Mathematical Problems in Engineering. 2015; 2015

[7] Fatihu Hamza Mukhtar, Jen Yap Hwa, Ahmed Choudhury Imtiaz. Cuckoo search algorithm based design of interval Type-2 Fuzzy PID Controller for Furuta pendulum system. Engineering Applications of Artificial Intelligence. 2017; 62

[8] Yang Xuebo, Zheng Xiaolong. Swing-Up and Stabilization Control Design for an Underactuated Rotary Inverted Pendulum System: Theory and Experiments. IEEE Transactions on Industrial Electronics. 2018; 65(9)

[9] Pujol-Vazquez Gisela, Acho Leonardo, Mobayen Saleh, Nápoles Amelia, Pérez Vega. Rotary inverted pendulum with magnetically external perturbations as a source of the pendulum's base navigation commands. Journal of the Franklin Institute. 2018; 355(10)

[10] Casanova Vicente, Alcaína José, Salt Julián, Pizá Ricardo, Cuenca Ángel. Control of the rotary inverted pendulum through threshold-based communication. ISA Transactions. 2016; 62

[11] Hamza Mukhtar Fatihu, Yap Hwa Jen, Choudhury Imtiaz Ahmed, Chiroma Haruna, Kumbasar Tufan. A survey on advancement of hybrid type 2 fuzzy sliding mode control. Neural Computing and Applications. 2017; 30(2)

[12] Hamza Mukhtar Fatihu, Yap Hwa Jen, Choudhury Imtiaz Ahmed, Isa Abdulbasid I, Zimit Aminu Y. Simulation Studies for Stabilization Control of Furuta Pendulum System Using Cascade Fuzzy PD Controller. International Journal of Simulation -. Systems, Science \& Technology. 2016; 17(35):1-9.

[13] Garćia-Alarćon Octavio, Puga-Guzman Sergio, Moreno-Valenzuela Javier. On parameter identification of the Furuta pendulum. Procedia Engineering. 2012; 35

[14] Chen Bing, Gao Shifan, Qu Yiming, Xu Nuo, Zhao Yi. An Euler-Lagrange Equation Oriented Solution for Write Energy Minimization of STT-MRAM. IEEE Transactions on Electron Devices. 2019; 66(8)

[15] Jabbar Abdul, Malik Fahad Mumtaz, Sheikh Shahzad Amin. Nonlinear stabilizing control of a rotary double inverted pendulum: a modified backstepping approach. Transactions of the Institute of Measurement and Control. 2016; 39(11)

[16] Isa Abdulbasid Ismail, Hamza Mukhtar F.. Effect of sampling time on PID controller design for a heat exchanger system. 2014 IEEE 6th International Conference on Adaptive Science \& Technology (ICAST). 2014.

[17] Eltag Khaled, Aslamx Muhammad Shamrooz, Ullah Rizwan. Dynamic Stability Enhancement Using Fuzzy PID Control Technology for Power System. International Journal of Control, Automation and Systems. 2019; 17(1)

[18] Magaji Nuraddeen, Hamza Mukhtar F., Dan-Isa Ado. Comparison of GA and LQR tuning of static VAR compensator for damping oscillations. International Journal of Advances in Engineering \& Technology. 2012; 2(1):594-601.

[19] Vinodh Kumar E., Jerome Jovitha. Robust LQR Controller Design for Stabilizing and Trajectory Tracking of Inverted Pendulum. Procedia Engineering. 2013; 64

[20] Zhou Kemin, Doyle John C., Glover Keith. Robust and optimal control. Prentice-Hall: New Jersey; 1996.

[21] Pandey Sumit Kumar, Laxmi Vijaya. Optimal Control of Twin Rotor MIMO System Using LQR Technique. Springer: New Delhi; 2015.

[22] Hamza Mukhtar Fatihu, Yap Hwa Jen, Choudhury Imtiaz Ahmed. Recent advances on the use of meta-heuristic optimization algorithms to optimize the type-2 fuzzy logic systems in intelligent control. Neural Computing and Applications. 2015; 28(5)

[23] Mendel Jerry M.. Advances in type-2 fuzzy sets and systems. Information Sciences. 2007; 177(1)

[24] Galichet S., Foulloy L.. Fuzzy controllers: synthesis and equivalences. IEEE Transactions on Fuzzy 
Systems. 1995; 3(2)

[25] Hu Bao-Gang, Mann G.K.I., Gosine R.G.. A systematic study of fuzzy PID controllers-function-based evaluation approach. IEEE Transactions on Fuzzy Systems. 2001; 9(5)

[26] Ghafouri Mohsen, Karaagac Ulas, Karimi Houshang, Jensen Simon, Mahseredjian Jean, Faried Sherif O.. An LQR Controller for Damping of Subsynchronous Interaction in DFIG-Based Wind Farms. IEEE Transactions on Power Systems. 2017; 32(6)

[27] Meshkov A.G., Sokolov V.V.. On third order integrable vector Hamiltonian equations. Journal of Geometry and Physics. 2017; 113 